\title{
Intraoperative Near-infrared Fluorescence Imaging with Novel Indocyanine Green-Loaded Nanocarrier for Spinal Metastasis: A Preliminary Animal Study
}

\author{
Toru Funayama, Masataka Sakane*, Tetsuya Abe, Isao Hara, Eiichi Ozeki and Naoyuki Ochiai \\ 1-1-1 Tennoudai, Tsukuba, Ibaraki, 305-8575, Japan/ Department of Orthopaedic Surgery, Graduate School of \\ Comprehensive Human Sciences, University of Tsukuba
}

\begin{abstract}
Marginal resection during resection of a spinal metastasis is frequently difficult because of the presence of important tissues such as the aorta, vena cava, and dura mater, including the spinal cord adjacent to the vertebral body. Thus, there is an urgent need for novel intraoperative imaging modalities with the ability to clearly identify bone metastasis. We have proposed a novel nanocarrier loaded with indocyanine green (ICG) (ICG-lactosome) with tumor selectivity attributable to its enhanced permeation and retention (EPR) effect. We studied its feasibility in intraoperative near-infrared (NIR) fluorescence diagnosis with ICG-lactosome for imaging spinal metastasis. A rat model of subcutaneous mammary tumor and a rat model of spinal metastasis of breast cancer were used. Fluorescence emitted by the subcutaneous tumors and the spinal metastasis were clearly detected for at least $24 \mathrm{~h}$. Moreover, imaging of the dissected spine revealed clear fluorescence emitted by the metastatic lesion in the L6 vertebra while the normal bone lacked fluorescence. This study was the first report on NIR fluorescence imaging of spinal metastasis in vivo. NIR fluorescence imaging with ICG-lactosome could be an effective intraoperative imaging modality for detecting spinal metastasis.
\end{abstract}

Keywords: Animal study, indocyanine green, intraoperative imaging modality, nanocarrier, near-infrared fluorescence imaging, spinal metastasis.

\section{INTRODUCTION}

Breast cancer is the most frequent site of origin of spinal metastasis [1, 2]. Previous studies reported that spinal metastasis would occur even if the primary lesion is controlled, and it is significantly associated with deterioration of the quality of life (QOL) of these patients due to pain and metastatic epidural spinal cord compression $[3,4]$. Although recent advances in surgery has improved the QOL of patients $[5,6]$, local recurrence is relatively common $[7,8]$ and the therapeutic options after recurrence are often limited to such as radiation therapy [7]. Therefore, it is important to control spinal metastasis, and there is an urgent need for novel intraoperative imaging modalities that are capable of identifying metastatic cancer tissue in bone.

Near-infrared (NIR) fluorescence optical imaging has become an increasingly attractive technology for the noninvasive diagnosis of diseases [9]. Recently, there has been considerable focus on the potential use of indocyanine green (ICG) in intraoperative real-time imaging [10-16]. However, the strong binding of plasma proteins with ICG and its subsequent rapid clearance by the liver results in binding of ICG to the tumor only for a short duration after administration; thus, the use of ICG for tumor imaging applications is limited [17].

\footnotetext{
*Address correspondence to this author at the 1-1-1 Tennoudai, Tsukuba, Ibaraki, 305-8575, Japan/ Department of Orthopaedic Surgery, Graduate School of Comprehensive Human Sciences, University of Tsukuba; Tel: +81-29-8533219; Fax: +81-29-8533214;

Emails: sakane-m@md.tsukuba.ac.jp, sakane.masataka@gmail.com
}

A potential approach to overcoming this disadvantage is delivering ICG in a nanocarrier that can provide increased stability and protection from nonspecific plasma protein binding and offer enhanced circulation time [18-20]. A novel nanocarrier, ICG-lactosome [21], which is a molecular assembly composed of hydrophobic poly(L-lactic acid) (PLLA) and hydrophilic poly(sarcosine) (PSar) amphiphilic block polydespsipeptide including ICG labeled PLLA in the hydrophobic inner core, provides good tumor imaging without accumulation to the liver. ICG-lactosome having a diameter of 30-40 $\mathrm{nm}$ shows high escape ability, stealth property in other word, from reticuloendothelial system (RES), and accumulated to the tumor region by the enhanced permeation and retention (EPR) effect [22]. This is because the high density of hydrophilic PSar chains so called polymer brush around the molecular assemblies contributes to the inhibition from RES recognition.

In this study, we aimed to investigate the usefulness of intraoperative NIR fluorescence imaging using ICGlactosome in the detection of spinal metastasis.

\section{MATERIALS AND METHODS}

\section{Tumor Cell Lines and Animal Care}

The rat mammary adenocarcinoma cell line CRL-1666 (also known as 13762 MAT B III) was selected and purchased from the American Type Culture Collection (Manassas, VA). The cells were cultured in Dulbecco's modified Eagle's medium (DMEM) containing 10\% fetal bovine serum, $80.5 \mathrm{pg} / \mathrm{ml}$ streptomycin, $80.5 \mathrm{U} / \mathrm{ml}$ penicillin, 
Table 1. Rats Examined in this Study

\begin{tabular}{|c|c|c|c|}
\hline Rat & Imaging Tissue & Day of Imaging After Cell/Tumor Implantation & Timing of Imaging After ICG-lactosome Injection \\
\hline \hline entry 1 & ST & 10 & control, 1min, 3, and 6 h \\
\hline entry 2 & ST & 10 & $12 \mathrm{~h}$ \\
\hline entry 3 & ST & 10 & $24 \mathrm{~h}$ \\
\hline entry 4 & ST & 10 & $48 \mathrm{~h}$ \\
\hline entry 5 & SM & 7 & $1,3,6$, and $12 \mathrm{~h}$ \\
\hline entry 6 & SM & 7 & $24 \mathrm{~h}$ \\
\hline entry 7 & SM & 11 & $24 \mathrm{~h}$ \\
\hline entry 8 & SM & 18 & $24 \mathrm{~h}$ \\
\hline
\end{tabular}

ST: subctaneous tumor

SM: spinal metastasis

and $1 \%$ L-glutamine, and maintained in the humidified atmosphere containing $5 \% \mathrm{CO}_{2}$ at a temperature at $37^{\circ} \mathrm{C}$. All animals were cared for in accordance with the guidelines of the Laboratory Animal Resource Center of University of Tsukuba. The rats were anesthetized by intraperitoneal injection of sodium pentobarbital (Somnopentil, $32.5 \mathrm{mg} / \mathrm{kg}$ body weight) before initiation of all the experiments.

\section{Materials and Instruments}

A chloroform solution $(1.0 \mathrm{ml})$ of PSar $_{70}-$ PLLA $_{30}(1.16$ $\mu \mathrm{mol})$ and ICG labeled PLLA 30 (17.4 nmol) was dripped into test tube. The solvent was removed under the reduced pressure and thin film was formed on wall surface of the tube. Ultrapure water $(3 \mathrm{ml})$ was added into the tube and the solution was incubated at 85 degrees $\mathrm{C}$ for 20 minutes. The resulting solution was lyophilized after filtration by $0.2 \mu \mathrm{m}$ pore size. The hydrodynamic diameter of ICG-lactosome was $38 \mathrm{~nm}$. Before imaging, the rats were intravenously injected with $0.5 \mathrm{ml}$ of $1 \mathrm{mg} / \mathrm{ml}$ ICG-lactosome solution (dissolved in saline) via the tail vein. The concentration of ICG was $1.93 \mu \mathrm{mol} / \mathrm{L}$. NIR fluorescence images were taken by Clairvivo OPT (Shimadzu Corporation, Kyoto, Japan). Four laser diodes at $785 \mathrm{~nm}$ with a total power of $1 \mathrm{~mW} / \mathrm{cm}^{2}$ for fluorescence excitation and a filter set (excitation at 785 $\mathrm{nm}$ and emission at $845 \mathrm{~nm}$ ) were used for imaging. Prior to imaging, the rats were anesthetized as described previously, and the hair of the lesion was cut using clippers.

\section{NIR Fluorescence Imaging of Subcutaneous Mammary Tumors}

We used four 8-week-old female Fischer-344 rats in this study. The rats received subcutaneous injection of $1.5 \times 10^{6}$ CRL-1666 cells near the right mammary gland. A subcutaneous tumor (approximately $1 \mathrm{~cm}$ in size) was created 10 days after the injection. One rat (No. 1) was imaged just before the ICG-lactosome injection (as a control) and at $1 \mathrm{~min}$ and 1,3 , and $6 \mathrm{~h}$ after the injection. The other 3 rats (No. 2, 3, and 4) were imaged 12, 24, and $48 \mathrm{~h}$ after the injection, respectively (Table 1). All the rats were placed in the supine position during imaging.

\section{NIR Fluorescence Imaging of Rat Spinal Metastasis of Breast Cancer}

We selected a rat model of spinal metastasis of breast cancer [23] for this investigation. Four female Fischer 344 rats (11-week-old) were used. A breast cancer section (1 mm $\times 1 \mathrm{~mm} \times 1 \mathrm{~mm})$ of CRL-1666 cells was implanted into a hole created in the L6 vertebral body of the rats [24]. Two rats were imaged at $1 \mathrm{~min}$ and 3, 6, 12 (No. 5), and $24 \mathrm{~h}$ (No. 6) after ICG-lactosome injection on day 7 after cell implantation. The remaining 2 rats with hind-limb paralysis (No. 7 and 8) were imaged on days 11 and 18, respectively (Table 1). All the rats were placed in the prone position during imaging. After the whole-body imaging procedure was completed, the animals were sacrificed and their lumbar spines were dissected, set in the supine position, and imaged again.

After the NIR fluorescence imaging, the dissected spines were fixed in $10 \%$ formalin solution, decalcified, and processed for hematoxylin and eosin (H\&E) staining. Axial cross-sections of the L6 vertebra were histologically inspected.

\section{RESULTS}

\section{NIR Fluorescence Imaging}

Fluorescence emitted at the subcutaneous tumor site (Fig. 1) was detected $1 \mathrm{~min}$ after the ICG-lactosome injection and gradually increased with time until $6 \mathrm{~h}$ (No. 1). Thereafter, the fluorescence intensity remained high until $24 \mathrm{~h}$ (No. 2 and 3). The fluorescence emitted from the tumor decreased, and was detected in the bladder at $48 \mathrm{~h}$ (No. 4). Fluorescence was also detected in hairless portions of the body such as the mouth, foot, and tail until $24 \mathrm{~h}$. Fluorescence of the lumbar spine (Fig. 2) was detected from 1 min after the ICGlactosome injection, and the fluorescence intensity remained high until $24 \mathrm{~h}$ (No. 5 and 6). Fluorescence intensity of the lumbar spine of the rats with hind-limb paralysis (No. 7 and 8) was also found to be high, without the presence of any other lesions. Imaging of the dissected spine revealed clear fluorescence emitted by the metastatic lesion in the L6 vertebra (Fig. 3).

\section{Histological Examination}

Histological examination revealed that the tumor developed into osteolytic lesions. Further, on day 7 after tumor implantation, the cancer cells had invaded the vertebral bone marrow spaces. On day 11 after implantation, the metastatic lesion destroyed the trabecular bone structures of the vertebra, invaded the spinal canal, and compressed the spinal cord (Fig. 3). 


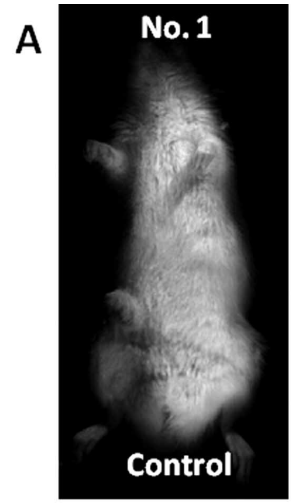

$\mathrm{E}$

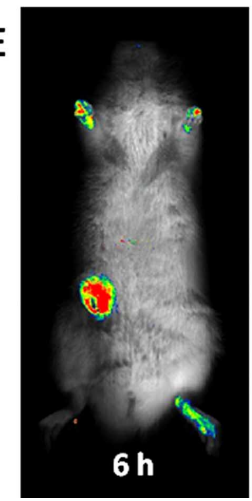

B

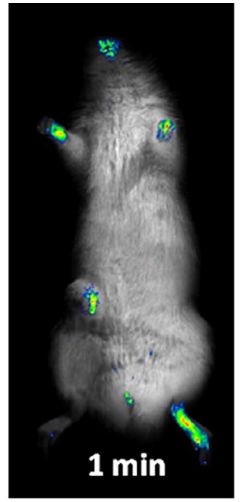

F

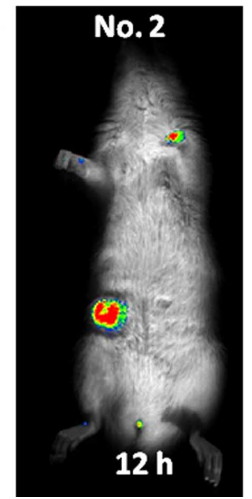

C

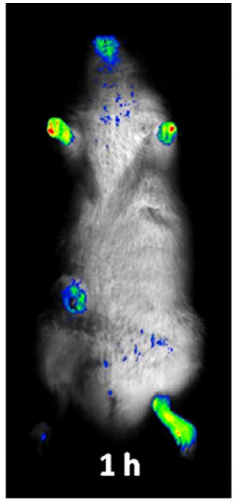

$\mathbf{G}$

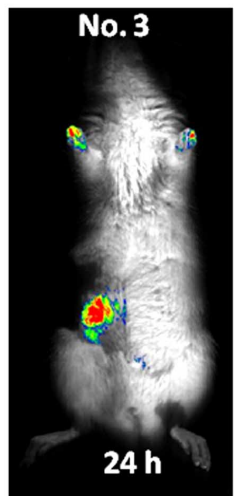

D

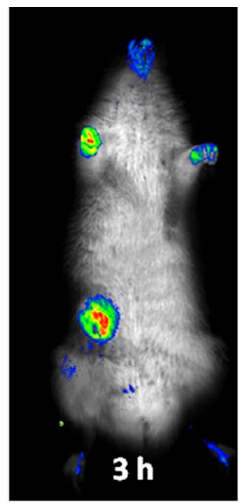

H

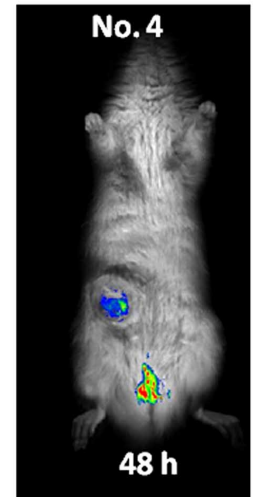

Fig. (1). Imaging for subcutaneous mammary tumor. No fluorescence was detected before the ICG-lactosome injection was administered (A). Fluorescence emitted by the subcutaneous tumor site detected at $1 \mathrm{~min}$ after the ICG-lactosome injection (B), gradually increased with time until $6 \mathrm{~h}(\mathbf{C}, \mathbf{D}$, and $\mathbf{E})$. Thereafter, the fluorescence intensity remained high until $24 \mathrm{~h}(\mathbf{F}$ and $\mathbf{G})$ and decreased at $48 \mathrm{~h}(\mathbf{H})$.
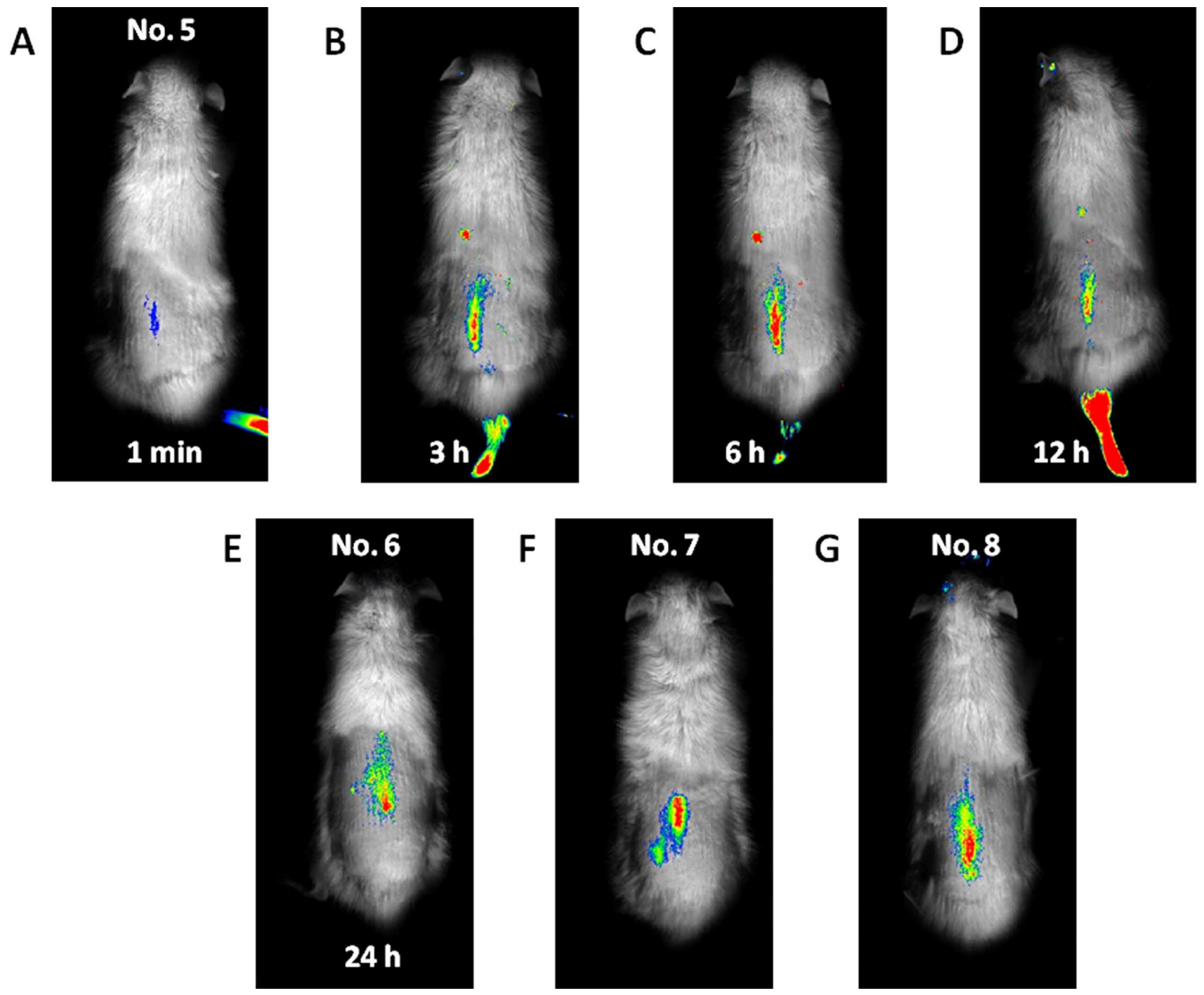

Fig. (2). Whole-body imaging for spinal metastasis of breast cancer. Fluorescence was first detected in the lumbar spine at 1 min after the ICG-lactosome injection (A), and its intensity remained high until $24 \mathrm{~h}(\mathbf{B}, \mathbf{C}, \mathbf{D}$, and $\mathbf{E})$. The fluorescence intensity in the lumbar spine of the rats with hind-limb paralysis was also high, and no other lesions were detected (F and $\mathbf{G})$. 

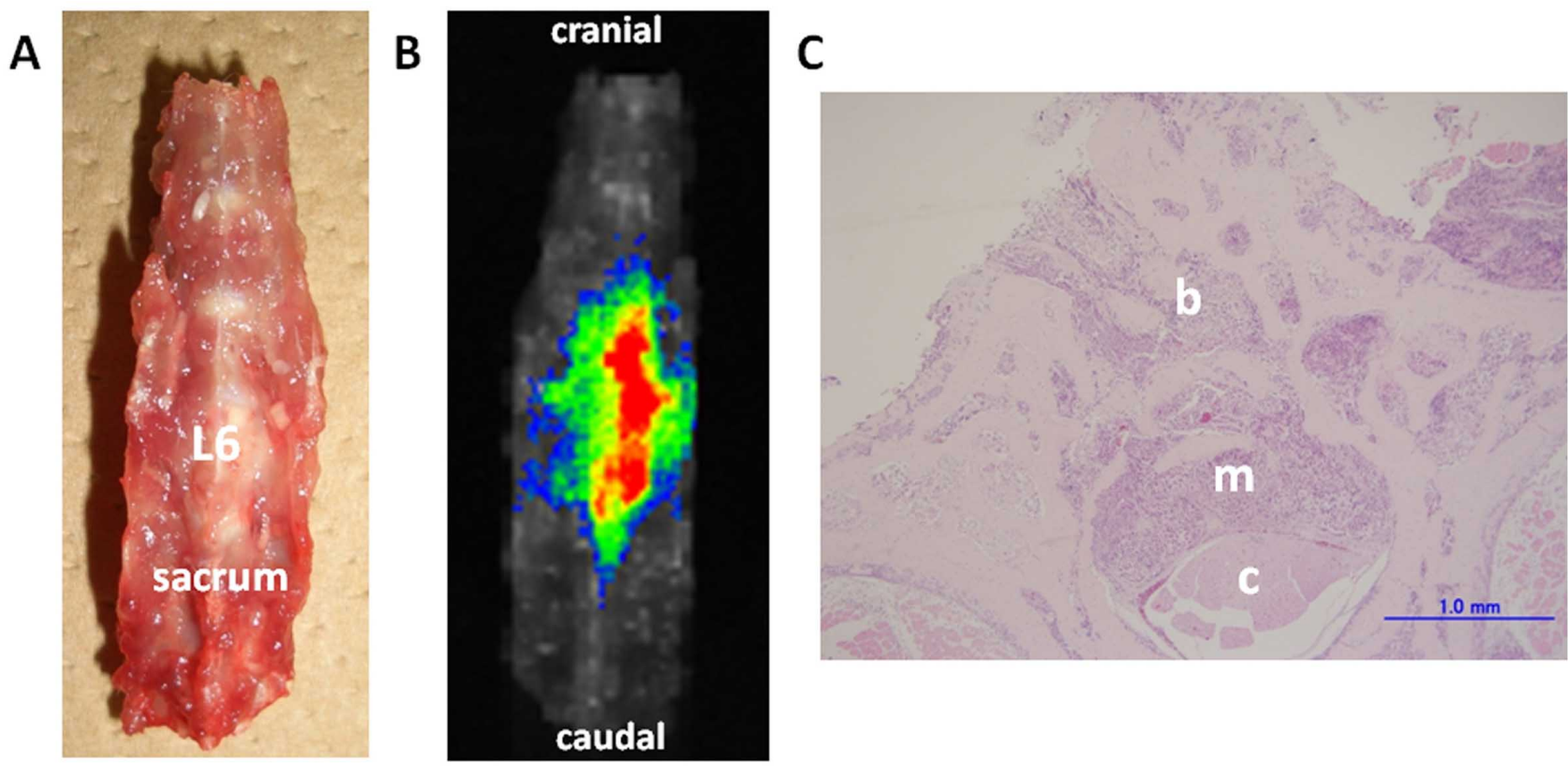

Fig. (3). Ex vivo imaging and histological examination of the dissected spine (Rat No. 7). The dissected lumbar spine was set in the supine position (A). Fluorescence can be clearly detected in the metastatic lesion in the L6 vertebra (B). Histological examination (C) of the axial cross-sections of the L6 vertebra (H\&E staining, scale bar $=1 \mathrm{~mm}$ ) shows invasion of the vertebral bone marrow spaces by cancer cells $(\mathbf{b})$. The metastatic lesion destroyed the trabecular bone structures of the spinal canal (m), and compressed the spinal cord (c) on day 11 after tumor implantation.

\section{DISCUSSION}

The results of this study revealed that NIR fluorescence imaging with the aid of ICG-lactosome could be used to selectively visualize spinal metastasis of breast cancer and subcutaneous mammary tumor. The fluorescence intensity of the lesions remained high for at least $24 \mathrm{~h}$ in all cases. This study was the first report on NIR fluorescence imaging of spinal metastasis in vivo. Further, we dissected the lumbar spine of the rats to investigate the efficacy of ex vivo NIR fluorescence imaging. The spinal metastasis lesion was clearly detected with fluorescence, and the normal bone lacked fluorescence and was easily identified.

In the process of bone metastasis, unique interactions are found between metastatic cancer cells and osteoclasts [25]. Since these interactions are critical to the development and progression of bone metastasis, a local treatment including control of the margin is very important. However, marginal resection during resection of a spinal metastasis is frequently difficult because of the presence of important tissues such as the aorta, vena cava, and dura mater, including the spinal cord adjacent to the vertebral body. Moreover, spinal metastasis is often associated with small skip lesions, which may be a cause of recurrence. Surgeon ought to rely on preoperative diagnostic modalities and intraoperative macroscopic inspection alone. Therefore, a novel intraoperative local imaging modality such as the one developed in the present study, which can clearly differentiate between metastasis and normal bone, could serve as a useful tool in the safe resection of spinal metastasis.

The NIR light excitation wavelength allows a penetration from approximately 2 to several centimeters into tissue [26, 27]. Thus, the NIR fluorescence imaging may be suitable for intraoperative using, while it is not appropriate for screening of human body. Since a CCD camera and a microscope with an ICG filter, which enabled visualization of the invisible near-infrared light, have been previously used in surgery [11, 12, 14-16], ICG-lactosome could function as a novel imaging probe, enabling the surgeon to easily diagnose the safety margin and small skip lesions intraoperatively.

The limitations of this study are as follows. (a) The control sample of the spinal metastasis model was not performed. We believe that no fluorescence should have been detected before the ICG-lactosome injection in the spinal metastasis model as well as the subcutaneous tumor model. However, the animal models were different between the subcutaneous model and the spinal metastasis model. This point should be considered in the future study. (b) The quantitative analysis data of the pathologic lesion and the specificity of ICG-lactosome to tumor tissues are unknown. Adequate quantitative analysis that is focused on these points should be investigated in the future studies.

Although further studies are needed, NIR fluorescence imaging for spinal metastasis using ICG-lactosome could be a useful intraoperative imaging modality. Moreover, ICG injection and NIR light irradiation have photodynamic effects on spinal metastasis [28]. Thus, the combination of ICG-lactosome and NIR light irradiation could be a novel therapeutic system, including the intraoperative imaging modality and photodynamic therapy.

\section{CONCLUSIONS}

We were able to selectively image spinal metastasis of breast cancer by NIR fluorescence imaging with the aid of ICG-lactosome. Although this is a preliminary study, our results demonstrate that imaging aided by ICG-lactosome could be a useful intraoperative imaging modality for spinal metastasis. 


\section{CONFLICT OF INTEREST}

No conflicting financial interests exist.

\section{ACKNOWLEDGEMENTS}

This study was partially supported by a grant from the Japan Science and Technology Agency, Innovation Satellite Ibaraki, and Shimadzu Corporation.

\section{REFERENCES}

[1] P. Bohm and J. Huber, "The surgical treatment of bony metastases of the spine and limbs," J. Bone Joint Surg. Br., vol. 84, no. 4, pp. 521-529, 2002.

[2] J. Schaberg and B. J. Gainor, "A profile of metastatic carcinoma of the spine," Spine (Phila Pa 1976), vol. 10, no. 1, pp. 19-20, 1985.

[3] J. L. Abrahm, M. B. Banffy, and M. B. Harris, "Spinal cord compression in patients with advanced metastatic cancer: "all I care about is walking and living my life"," JAMA, vol. 299, no. 8, pp. 937-946, 2008.

[4] H. Oka, T. Kondoh, A. Seichi, T. Hozumi, and K. Nakamura, "Incidence and prognostic factors of Japanese breast cancer patients with bone metastasis," J. Orthop. Sci., vol. 11, no. 1, pp. 13-19, 2006.

[5] A. Ibrahim, A. Crockard, P. Antonietti, S. Boriani, C. Bunger, A. Gasbarrini, A. Grejs, J. Harms, N. Kawahara, C. Mazel, R. Melcher, and K. Tomita, "Does spinal surgery improve the quality of life for those with extradural (spinal) osseous metastases? An international multicenter prospective observational study of 223 patients. Invited submission from the Joint Section Meeting on Disorders of the Spine and Peripheral Nerves, March 2007," $J$. Neurosurg. Spine, vol. 8, no. 3, pp. 271-278, 2008.

[6] R. A. Patchell, P. A. Tibbs, W. F. Regine, R. Payne, S. Saris, R. J. Kryscio, M. Mohiuddin, and B. Young, "Direct decompressive surgical resection in the treatment of spinal cord compression caused by metastatic cancer: a randomised trial," Lancet, vol. 366, no. 9486, pp. 643-648, 2005.

[7] J. S. Cole and R. A. Patchell, "Metastatic epidural spinal cord compression," Lancet Neurol., vol. 7, no. 5, pp. 459-466, 2008.

[8] H. Sakaura, N. Hosono, Y. Mukai, T. Ishii, K. Yonenobu, and H. Yoshikawa, "Outcome of total en bloc spondylectomy for solitary metastasis of the thoracolumbar spine," J. Spinal Disord. Tech., vol. 17, no. 4, pp. 297-300, 2004.

[9] M. C. Pierce, D. J. Javier, and R. Richards-Kortum, "Optical contrast agents and imaging systems for detection and diagnosis of cancer," Int. J. Cancer, vol. 123, no. 9, pp. 1979-1990, 2008.

[10] B. E. Schaafsma, J. S. Mieog, M. Hutteman, J. R. van der Vorst, P. J. Kuppen, C. W. Lowik, J. V. Frangioni, C. J. van de Velde, and A. L. Vahrmeijer, "The clinical use of indocyanine green as a nearinfrared fluorescent contrast agent for image-guided oncologic surgery," J. Surg. Oncol., vol. 104, no. 3, pp. 323-32.

[11] G. P. Colby, A. L. Coon, D. M. Sciubba, A. Bydon, P. Gailloud, and R. J. Tamargo, "Intraoperative indocyanine green angiography for obliteration of a spinal dural arteriovenous fistula," $J$. Neurosurg. Spine, vol. 11, no. 6, pp. 705-709, 2009.

[12] T. Handa, R. G. Katare, H. Nishimori, S. Wariishi, T. Fukutomi, M. Yamamoto, S. Sasaguri, and T. Sato, "New device for intraoperative graft assessment: HyperEye charge-coupled device camera system," General Thorac Cardiovasc. Surg, vol. 58, no. 2, pp. 68-77, 2010.

[13] M. V. Marshall, J. C. Rasmussen, I.-C. Tan, M. B. Aldrich, K. E. Adams, X. Wang, C. E. Fife, E. A. Maus, L. A. Smith, and E. M. Sevick-Muraca, "Near-infrared fluorescence imaging in humans with indocyanine green: a review and update," J. Surg Oncol., vol. 2, no. pp. 12-25, 2010.
[14] J. K. Oh, H. C. Shin, T. Y. Kim, G. H. Choi, G. Y. Ji, S. Yi, Y. Ha, K. N. Kim, and D. H. Yoon, "Intraoperative indocyanine green video-angiography: spinal dural arteriovenous fistula," Spine, [Epub Ahead of print].

[15] G. A. Schubert, M. Barth, and C. Thome, "The Use of Indocyanine Green Videography for Intraoperative Localization of Intradural Spinal Tumors," Spine, 2010 [E-pub Ahead of print].

[16] G. A. Schubert, K. Schmieder, M. Seiz-Rosenhagen, and C. Thome, "ICG Videography Facilitates Interpretation of Vascular Supply and Anatomical Landmarks in Intramedullary Spinal Lesions: Two Case Reports," Spine, vol. 36, no. 12, pp. E811-813, 2011.

[17] K. Licha, B. Riefke, V. Ntziachristos, A. Becker, B. Chance, and W. Semmler, "Hydrophilic cyanine dyes as contrast agents for near-infrared tumor imaging: synthesis, photophysical properties and spectroscopic in vivo characterization," Photochem. Photobiol., vol. 72, no. 3, pp. 392-398, 2000.

[18] E. I. Altinoglu, T. J. Russin, J. M. Kaiser, B. M. Barth, P. C. Eklund, M. Kester, and J. H. Adair, "Near-infrared emitting fluorophore-doped calcium phosphate nanoparticles for in vivo imaging of human breast cancer," ACS Nano., vol. 2, no. 10, pp. 2075-2084, 2008.

[19] T. H. Kim, Y. Chen, C. W. Mount, W. R. Gombotz, X. Li, and S. H. Pun, "Evaluation of temperature-sensitive, indocyanine greenencapsulating micelles for noninvasive near-infrared tumor imaging," Pharm. Res., vol. 27, no. 9, pp. 1900-1913, 2010.

[20] H. Tanisaka, S. Kizaka-Kondoh, A. Makino, S. Tanaka, M. Hiraoka, and S. Kimura, "Near-infrared fluorescent labeled peptosome for application to cancer imaging," Bioconjug. Chem., vol. 19, no. 1, pp. 109-117, 2008.

[21] A. Makino, S. Kizaka-Kondoh, R. Yamahara, I. Hara, T. Kanzaki, E. Ozeki, M. Hiraoka, and S. Kimura, "Near-infrared fluorescence tumor imaging using nanocarrier composed of poly(L-lactic acid)block-poly(sarcosine) amphiphilic polydepsipeptide," Biomaterials, vol. 30, no. 28, pp. 5156-5160, 2009.

[22] Y. Matsumura and H. Maeda, "A new concept for macromolecular therapeutics in cancer chemotherapy: mechanism of tumoritropic accumulation of proteins and the antitumor agent smancs," Cancer Res., vol. 46, no. 12 Pt 1, pp. 6387-6392, 1986.

[23] A. Mantha, F. G. Legnani, C. A. Bagley, G. L. Gallia, I. Garonzik, G. Pradilla, E. Amundson, B. M. Tyler, H. Brem, and Z. L. Gokaslan, "A novel rat model for the study of intraosseous metastatic spine cancer," J. Neurosurg. Spine, vol. 2, no. 3, pp. 303-307, 2005

[24] T. Abe, M. Sakane, T. Ikoma, M. Kobayashi, S. Nakamura, and N. Ochiai, "Intraosseous delivery of paclitaxel-loaded hydroxyapatitealginate composite beads delaying paralysis caused by metastatic spine cancer in rats," J. Neurosurg. Spine, vol. 9, no. 5, pp. 502-510, 2008.

[25] T. Yoneda and T. Hiraga, "Crosstalk between cancer cells and bone microenvironment in bone metastasis," Biochem. Biophys. Res. Commun., vol. 328, no. 3, pp. 679-687, 2005.

[26] M. Mitsunaga, M. Ogawa, N. Kosaka, L. T. Rosenblum, P. L. Choyke, and H. Kobayashi, "Cancer cell-selective in vivo near infrared photoimmunotherapy targeting specific membrane molecules," Nat. Med., vol. 17, no. 12, pp. 1685-1691, 2011.

[27] J. R. Starkey, A. K. Rebane, M. A. Drobizhev, F. Meng, A. Gong, A. Elliott, K. McInnerney, and C. W. Spangler, "New two-photon activated photodynamic therapy sensitizers induce xenograft tumor regressions after near-IR laser treatment through the body of the host mouse," Clin. Cancer Res., vol. 14, no. 20, pp. 6564-6573, 2008.

[28] T. Funayama, M. Sakane, T. Abe, and N. Ochiai, "Photodynamic therapy with indocyanine green injection and near-infrared light irradiation has phototoxic effects and delays paralysis in spinal metastasis," Photomed. Laser Surg., vol. 30, no. 1, pp. 47-53, 2012.

(C) Funayama et al.; Licensee Bentham Open.

This is an open access article licensed under the terms of the Creative Commons Attribution Non-Commercial License

(http://creativecommons.org/licenses/by-nc/3.0/) which permits unrestricted, non-commercial use, distribution and reproduction in any medium, provided the work is properly cited. 